Article

\title{
Annualization of Renewable Investment Costs for Finite Horizon Electricity Pricing and Cost Recovery
}

\author{
Fco. Alberto Campos ${ }^{1, *}$, José Villar ${ }^{2}(\mathbb{D})$ and Efraim Centeno ${ }^{1}(\mathbb{D}$ \\ 1 Institute for Research in Technology, Technical School of Engineering, Comillas Pontifical University, \\ 28015 Madrid, Spain; efraim.centeno@comillas.edu \\ 2 Institute for Systems and Computer Engineering, Technology and Science, 465 Porto, Portugal; \\ jose.villar@inesctec.pt \\ * Correspondence: acampos@comillas.edu
}

Citation: Campos, F..A.; Villar, J.; Centeno, E. Annualization of Renewable Investment Costs for Finite Horizon Electricity Pricing and Cost Recovery. Sustainability 2021, 13, 1993. https://doi.org/10.3390/ su13041993

Academic Editor: Donato Morea

Received: 17 December 2020

Accepted: 5 February 2021

Published: 12 February 2021

Publisher's Note: MDPI stays neutral with regard to jurisdictional claims in published maps and institutional affiliations.

Copyright: (c) 2021 by the authors. Licensee MDPI, Basel, Switzerland. This article is an open access article distributed under the terms and conditions of the Creative Commons Attribution (CC BY) license (https:// creativecommons.org/licenses/by/ $4.0 /)$.

\begin{abstract}
The increasing penetration of renewable electricity generation is complicating the bidding and estimating processes of electricity prices, partly due to the shift of the overall cost sensitivity from operation (fuel) costs to investment costs. However, cost minimization models for capacity expansion are frequently based on the principle that, for a perfectly adapted system allowing non-served energy, marginal remuneration allows overall operation and investments costs recovery. In addition, these models are usually formulated as finite-horizon problems when they should be theoretically solved for infinite horizons under the assumption of companies' infinite lifespan, but infinite horizon cannot be dealt with mathematical programming since it requires finite sets. Previous approaches have tried to overcome this drawback with finite horizon models that tend asymptotically to the original infinite ones and, in many cases, the investment costs are annualized based on the plants' lifespan, sometimes including a cost residual value. This paper proposes a novel approach with a finite horizon that guarantees the investment costs' recovery. It is also able to obtain the marginal electricity costs of the original infinite horizon model, without the need for residual values or non-served energy. This new approach is especially suited for long-term electricity pricing with investments in renewable assets when non-served demand is banned or when no explicit capacity remuneration mechanisms are considered.
\end{abstract}

Keywords: infinite horizon models; investment theory; marginal pricing; renewable technologies

JEL Classification: G12; O21; Q55; L94; C61

\section{Introduction \\ 1.1. Problem Description}

In 2030, half of the EU's electricity demand will most likely be met with renewable energy sources, and by 2050 the power sector will have to be almost fully renewable. Classical marginalist theory [1] states that assuming: (1) marginal cost pricing based on short-term marginal production costs; (2) a perfectly adapted system; (3) a set of technologies available for investments; and (4) a cost for the non-supplied energy, the overall cost (investment plus operational costs) is recovered for all technologies. However, this theory requires the existence of unsupplied demand at certain peak hours with very high prices, in order to allow marginal plants to recover their overall costs, which is unrealistic from a practical point of view in many power systems in developed countries. Moreover, current market mechanisms based on the marginalist theory could not provide adequate economic signals for investment, especially in systems with a high share of renewables. Therefore, remuneration mechanisms for generation, based only on short-term variable costs could imperil the 2050 EU objectives. Indeed, in practice, null variable costs of renewables lead to close-to-zero prices, making it more difficult to recover their full costs, thus compromising not only their profitability but also the economic viability of other backup technologies 
needed to meet demand in the absence of wind and sun and to provide regulation reserves. Additionally, the integration of renewable installations might lead to systems that suffer from an effect of profitability-loss cannibalization, since the higher the renewable production, the lower the market incomes received by all generation assets [2].

In this context, well-designed capacity remuneration mechanisms may overcome this problem, as capacity pricing can establish the amount of firm capacity that can be provided by each technology, being an essential tool for cost-effective capacity investments. The absence of these mechanisms may discourage capacity providers from investing in new generation capacity, putting at risk supply security.

This paper analyzes the problem of investment cost recovery in a power system with a large penetration of renewables without the existence of an explicit capacity remuneration mechanism. This is a critical problem for future fully renewable systems even with a capacity remuneration mechanism since, as discussed in [3], renewable generation has so far had very limited participation in capacity mechanisms.

\subsection{State of the Art}

Generation expansion studies used to analyze the problem of cost recovery in the absence of explicit capacity mechanisms are frequently based on the formulation of finitehorizon mathematical programming (MP) problems, even knowing that finite horizons are not suitable in the long-term planning with infinite-life generation companies, [4]. Two main approaches to overcome this problem have been proposed in the literature on this topic, the static and the dynamic approaches [5].

\subsubsection{The Static Approach}

This approach determines the optimal investment plant (OIP) for a single representative year in the future, dividing the investment costs by the lifespan of the generation assets. Classic works that use this approach are [6-10]. For example, static MP models including the network constraints are presented in $[9,10]$. A more recent static cost minimization MP model including the network is described in [11], while [12,13] present static MP models for the analysis of the operating reserves and the carbon dioxide emissions, respectively. While it is useful in some situations for simplified studies, this single-year static approach may be misleading in the current context of the rapid development of renewable energy technologies, as their investment costs decrease on a yearly basis. Static analyses provide very vague results regarding the year in which investments should be made since they do not provide insights on how electricity prices could evolve annually.

\subsubsection{The Dynamic Approach}

Unlike the static approach, the dynamic approach computes the OIP for a multi-year finite horizon. A residual value modeling the costs for the years beyond the finite horizon is usually included indefinitely replicating the cost of the last year of the horizon [14]. A residual value is alternatively considered in [15], which uses the resale value of the generation assets at the end of the study year. However, residual values may be volatile and complex to compute when considering a company with a big market share. Moreover, the replication may lead to inaccurate results, for example, if investment costs decrease significantly over time, as commonly happens with renewable technologies. Some other works such as [16-19], do not represent residual values, assuming that they can be disregarded under some circumstances such as, for example, for long finite horizons and/or high discount rate values.

Most of the above works distribute overall costs over the years by applying a particular annualization of these costs and allocating them based on the generation assets' lifespan. The most classical annualization of costs is based on the capital recovery factor. For example, in [20] a capital recovery annualization is applied to reduce renewable energy curtailment by improving the accommodation of this generation taking into account 
short-term operation constraints and uncertainties from the load and renewable energy sources.

\subsection{Contributions of the Proposed Dynamic Approach}

This paper overcomes some of the limitations of the abovementioned approaches by analyzing the problem of determining the long-term marginal costs (hereinafter termed electricity prices, for simplicity's sake) in order to guarantee the recovering of the investment costs of renewable technologies. It applies a dynamic approach, more suitable than the described static ones, in the absence of an explicit capacity mechanism, and when non-served demand is not allowed. Unlike the dynamic approaches presented in the literature, this research:

- Focuses on the long-term recovering electricity prices (understood as those that, under some assumptions, guarantee the recovery of the total costs of producing electricity), computed by the dual variables of the generation-demand balance constraints of the MP problems, instead of aiming at the primal variables to estimate the OIP as other approaches do (for instance [16-19]).

- Assuming infinite-life generation companies, proposes a finite MP problem with a novel annualization of the investment costs which ensures that the electricity prices obtained from the finite problem are the same as those obtained using the corresponding infinite model. As far as the authors know, previous works (for instance [15]) have not been able to ensure the fulfilment of this interesting and relevant property. Hereinafter, when this happens, it will be said that the annualization method verifies the finite dual pricing (FDP) property. More specifically, if $C^{\infty}$ is the overall cost of the model for an OIP under an infinite horizon, this paper formulates a finite truncation $C$ of $C^{\infty}$ using only $p$ years such that the derivatives of $C$ and $C^{\infty}$ with respect to the demand $d_{p}$ at any year $p \leq p$ (the dual variables of the generation-demand balance constraint) coincide. This paper also proves the well-known result that states that the straightforward truncation $C^{p}$ of $C^{\infty}$ (i.e., solving the problem for a finite number of years without annualization) does not verify the FDP property and leads to large runtimes when demanding a reasonable approximation of $C^{\infty}$ by $C^{p}$.

- Formulates, in order to deduce the annualization method, a stylized cost-minimization MP problem with a finite horizon without the need of considering the abovementioned residual value that current dynamic approaches need (for instance $[14,15])$, which makes it easier both from a theoretical and practical point of view.

- Significantly reduces the estimation time needed to reach reasonable approximations of the electricity prices relative to other dynamic approaches based on finite MP problems (for instance [21]), due to the contribution referred in the previous points.

- Even if the annualization proposed guarantees the total cost recovery only under some assumptions (see Section 2), in view of the lack of methods that guarantee the FDP property, reasonable price estimations could be obtained even under more relaxed hypothesis. However, additional research is needed in this point.

In short, this paper presents a relevant theoretical advance that allows simplifying an infinite OIP problem into a finite one to estimate long term electricity prices. This approach can be directly used from a practical point of view to estimate the total electricity price that guarantees investments' recovery for the economic sustainability of the power sector. This result is useful both for the investment planning of the electricity companies and for the regulators assessment of current markets designs.

This paper is structured as follows. The main assumptions that define the presented framework are described in the following section. Section 3 is devoted to analyzing the OIP and its sensitivity with respect to the demand. From this result, the marginal prices that guarantee the investment costs recovery are computed in Section 4, including and excluding corporate taxes. Section 5 presents the annualized investment costs proposed in this paper and proves how it fulfils the FDP property. Conclusions and future developments are discussed in the final section. 


\section{Main Assumptions}

The main assumptions of this paper are: (1) for simplicity's sake, investments are made in renewable generation with null operational costs; (2) no capacity remuneration mechanisms are explicitly modeled nor needed; (3) no unsupplied demand is allowed, as occurs in many real systems; and (4) other remuneration mechanisms might not exist.

The following modeling hypotheses have been also assumed (parameters are written in lowercase, decision variables in uppercase, and sets with capital Greek letters):

1. There is a centralized decision-maker with infinite life that minimizes the total investment costs of generation assets with null operation costs.

2. A finite temporal horizon $\Gamma \subseteq \mathbb{Z}$, which is discretized into periods $p$ whose duration is typically one year for long-term planning is considered. Periods $p \in \mathbb{N}$ are those where investments $K_{p}$ (in MW) must be decided, while $K_{p}$ for $p \in \Gamma \backslash \mathbb{N}$ are investments that have already been decided (and, therefore, they are inputs, i.e., $K_{p}=k_{p}$ ).

3. The lifespan of the investment $K_{p}$ at period $p$ is $l>1$, i.e., the lifespan extends from $p$ to $p+l-1$.

4. The rate $r_{p}>0$ to discount a future amount of money to reflect its present value does not depend on $p$, i.e., $r_{p}=r$.

5. Only one technology available for investment and, as other authors assume (see for example [22]), its investment cost $f_{p}(€ / \mathrm{MW} /$ period) increases according to inflation $i$ :

$$
f_{p+p^{\prime}}=f_{p} \cdot(1+i)^{p^{\prime}}=f_{0} \cdot(1+i)^{p^{\prime}+p}
$$

6. The utilization $e_{p}$ of capacity $K_{p}$ does not depend on $p$, i.e., $e_{p}=e$. Parameter $e$ is well-known as the utilization of the asset $K_{p}$, i.e., the ratio of the energy produced in $p$ divided by the installed power. Therefore, a mature technology is assumed.

7. Inflation $i$ is less than $r$ and, therefore, the present value of the investment $\operatorname{cost} f_{p}$ is decreasing:

$$
\frac{f_{p+1}}{(1+r)}=\left(\frac{1+i}{1+r}\right) \cdot f_{p}<f_{p}
$$

Hereinafter the quotient $(1+i) /(1+r)$ will be called real discount factor (RDF).

8. Demand $d_{p}$ (MWh demanded in $p$ ) is inelastic and must always be satisfied. It is represented as a single block per year that, in capacity expansion analyses, may be inputted as the yearly peak value multiplied by the number of hours in $p$. Note that $d_{p}$ must be satisfied with the capacities $K_{p^{\prime}}$ at periods $p^{\prime} \in \Gamma$ with $p-l+1 \leq p^{\prime} \leq p$.

Although the hypotheses described here may appear simplistic (for example, the consideration of only one technology for investment), from a practical point of view, the annualization of the resulting investment costs, based on a thorough and theoretical rigorous mathematical analysis, must be understood as a mathematical financial tool for investment decisions that may be applied in more complex analyses (like those with more than one technology for investment). Indeed, several annualizations may be chosen but the proposed one guarantees the FDP property under the abovementioned hypotheses, which could be relaxed in the absence of more rigorous studies. Understood in this way, the annualization we propose is a powerful mathematical tool to estimate long term electricity prices, assuming that, whatever the market mechanism is, total electricity prices should guarantee the investment costs recovery, ensuring the economic sustainability of the power sector even when non-served demand is not allowed. Moreover, the proposed annualization can help not only in the investment planning of electricity companies, but also in the reforming processes of current electricity markets. These processes must be set by regulators, which need tools to assess, for instance, the need of capacity remuneration mechanisms in power systems with a large amount of renewable generation, in which energy only markets tend to fail due to the existence of very low variable costs. 


\section{Optimal Investment Plan (OIP)}

Considering the above-mentioned assumptions, this section describes some useful properties of the OIP and how to obtain it.

\subsection{OIP Computation}

Appendix A proves that the OIP is unique and can be directly computed as follows:

$$
K_{p}=k_{p-m_{p} \cdot l}+\frac{d_{p-\left(m_{p}-1\right) \cdot l}-d_{p-\left(m_{p}-1\right) \cdot l-1}}{e}+\ldots+\frac{d_{p-l}-d_{p-l-1}}{e}+\frac{d_{p}-d_{p-1}}{e}
$$

where:

$$
m_{p}=\min \{m \in \mathbb{N} / p-m \cdot l<0\}
$$

Therefore, the new capacity investments $K_{p}$ at period $p$ depend only on inputs of the model, namely, the demand in periods before or equal to $p$, the hours of utilization $e$, and the capacity $k_{p^{\prime}}$ already installed for the period $p^{\prime} \in \Gamma \backslash \mathbb{N}$ closest to the first period $(p=0)$ of the temporal horizon. Note that each quotient in (3) represents the capacity needed to satisfy the variation of the demand in two consecutive years $p^{\prime}$ and $p^{\prime}-1, p^{\prime}$ being a year before $p$ a number of times multiple of the lifespan $l$, when certain quantity of the installed capacity expires.

\subsection{OIP Sensitivity with Respect to the Demand}

Appendix B proves that the variation $\Delta K_{p^{\prime}}$ of the OIP with respect to the variation $\Delta D_{p}$ in the demand $d_{p}$ at period $p$ is:

$$
\frac{\Delta K_{p^{\prime}}}{\Delta D_{p}}=\left\{\begin{array}{c}
\frac{1}{e} \text { if } p^{\prime}=p+n \cdot l \\
-\frac{1}{e} \text { if } p^{\prime}=p+n \cdot l+1 \text { for } n \in \mathbb{N} \\
0 \text { otherwise }
\end{array}\right.
$$

This result will be used in Section 5 to analyze how $\Delta D_{p}$ influences the total cost of the investments and means that the variation $\Delta K_{p^{\prime}}$ in a future period $p^{\prime}$ respect to the variation $\Delta D_{p}$ in a past period $p$ takes only values if the difference between both periods $p^{\prime}$ and $p$ is a multiple of the lifespan $l$ or a multiple of $l$ plus 1 (since, as mentioned before, the capacity $K_{p^{\prime}}$ must satisfy the variation of the demand in two previous consecutive years $p$ and $p-1$ ). Moreover, when positive, the variation $\Delta D_{p}$ relative to $\Delta K_{p}$ is the utilization $e$ or $-e$ since $d_{p}-d_{p}-{ }_{1}$ is not satisfied with the OIP but with the production generated from the OIP.

\section{Prices to Recover Investment Costs}

Appendix $C$ proves that, for the previously computed OIP, the energy prices $P_{p}$ $(€ / \mathrm{MWh})$ that allow the exact recovery of the investment costs $f_{p}$ satisfy:

$$
P_{p}=\frac{f_{p}}{e \cdot s}=P_{0} \cdot(1+i)^{p}
$$

where:

$$
s=\sum_{p=0}^{l-1}\left(\frac{1+i}{1+r}\right)^{p}
$$

Therefore, on the one hand, price $P_{p}$ is equal to $f_{p}$ divided by the utilization $e$ of the investment, taking into account $s$, which is the summation of RDF over the generation assets' lifespan. On the other hand, Equation (6) identifies the well-known result that states that the prices to recover investment costs growing up with the inflation $i$ should also increase with the inflation $i$ [23]. Taxes can be taken into account in (6) by substituting $f_{p}$ with $f_{p}^{\prime}$ as shown in Appendix D. 


\section{Finite Dual Pricing Property (FDP)}

This section presents the annualized investment costs proposed in this paper and proves how the resulting electricity marginal prices fulfils the FDP property. It also presents how these prices can be computed with MP, avoiding complexities like dealing with infinite horizons.

\subsection{The Infinite Horizon Problem with Cost $C^{\infty}$ : Prices to Recover Investment Costs as Dual Variables}

If the original infinite horizon problem is considered, the minimization of the present value of the total investment cost is:

$$
\underset{K_{p} \geq 0}{\operatorname{Min}}\left\{C^{\infty}=\sum_{p^{\prime}=0}^{\infty} \frac{f_{p^{\prime}} \cdot K_{p^{\prime}}}{(1+r)^{p^{\prime}}}\right\}
$$

subject to the generation-demand balance constrain $\left(d_{p}\right.$ must be satisfied with a utilization $e$ of the non-expired investments $K_{p^{\prime}}$ installed at periods before $p$, i.e., $p-l+1 \leq p^{\prime} \leq p$ ):

$$
d_{p}=e \cdot \sum_{p^{\prime}=p-l+1}^{p} K_{p^{\prime}}
$$

Appendix E proves that the dual variables $L_{p}^{\infty}$ of (9) when minimizing the objective function in (8) are:

$$
L_{p}^{\infty}=\left\{\begin{array}{c}
\sum_{n \in\{\mathbb{N}: p+n \cdot l \leq \infty\}} \frac{f_{0}}{e} \cdot\left(\frac{1+i}{1+r}\right)^{p+n \cdot l} \\
\sum_{n \in\{\mathbb{N}: p+n \cdot l+1 \leq \infty\}} \frac{f_{0}}{e} \cdot\left(\frac{1+i}{1+r}\right)^{p+n \cdot l+1}
\end{array}\right\}
$$

It also proves that $L_{p}^{\infty}$ are the discounted prices $P_{p}$ of (6) (taxes can be considered as indicated in Appendix D), i.e.,:

$$
L_{p}=\frac{P_{p}}{(1+r)^{p}}
$$

This result can be also drawn from [23]. However, $C^{\infty}$ has an infinite summation that makes impossible the exact computation of primal investment decisions with MP. To overcome this drawback, the next subsection presents a finite truncation $C$ of $C^{\infty}$ that guarantees the FDP property without a residual value in its formulation.

\subsection{The Straightforward Truncation $C^{p}$ of $C^{\infty}$}

It is well known that the straightforward truncation $C^{p}$ of $C^{\infty}$ up to $p$ periods $\left(C^{\infty}\right.$ with $\infty=p$ in (8)), without including a residual value in $C^{p}$, is not valid for computing the discounted prices $P_{p}$ as the dual variables $L_{-p}^{p}$ of (9) minimizing $C^{p}$ (note that $C^{p}$ and $L^{p} p$ are formulated as $C^{\infty}$ and $L^{\infty}{ }_{p}$ substituting $\infty$ by $\underline{p}$ in (8) and (10), respectively). As an example, let us consider the data in Table 1.

Table 1. Inputs of the case example.

\begin{tabular}{ccccc}
\hline$f_{0}(\boldsymbol{\epsilon} / \mathrm{MW} /$ Year) & $\boldsymbol{l}$ (Years) & $\boldsymbol{e}$ (Hours) & $\boldsymbol{i}(\%)$ & $\boldsymbol{r}(\%)$ \\
\hline $1.4 \cdot 10^{6}$ & 5 & 2193 & 2 & 10 \\
\hline
\end{tabular}

Apart from the chosen lifespan $l=5$ which has been kept small for the sake of simplicity (even though this will yield too high energy prices in this example), the rest of the data may 
correspond to onshore wind farm installations (according to the International Renewable Energy Agency, see [24]). With these data, (7) leads to $s=4.3237$.

Using this dataset, Figure 1 depicts $L_{-7}^{p}$ (green discontinuous line named by LS7) and the discounted price $P_{7} /(1+r)^{7}=87.03 € / \mathrm{MWh}$ (brown line named by P7), as a function of $p$. Each grey column in Figure 1 represents one of the two summations in (10) (making $\infty \equiv p$ ), positive columns (grey columns named by $\mathrm{P}$ ) corresponding to the first summation (adding $n \in \mathbb{N}$ such that $p+n \cdot 5 \leq p$, with $p=7$ ) while negative ones (grey columns named by $\mathrm{N}$ ) to the second $(n \in \mathbb{N}: 7+n \cdot \overline{5}+1 \leq p)$. According to (10), $L_{-7}^{p}$ is the summation of all the columns (whether positive or negative ones) before $\underline{p}$.

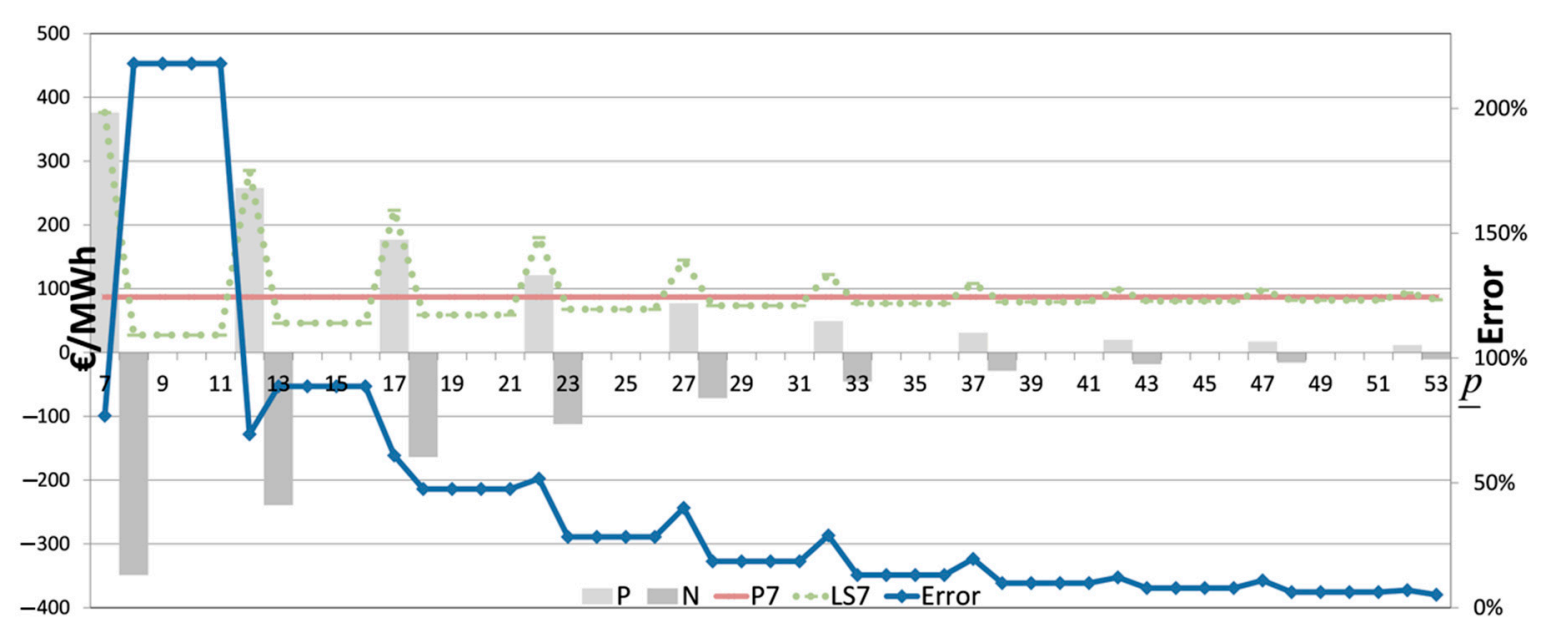

Figure 1. Approximation error of $\mathrm{C}^{\infty}$ for finite dual pricing (FDP).

Note that for any $\underline{p}, L_{-7}^{p}$ does not exactly coincide with $P_{7} /(1+r)^{7}$. However, a good approximation can be computed with a value of $p$ sufficiently large. Figure 1 also presents the approximation error of $L_{-7}^{p}$ with respect to $P_{7} /(1+r)^{7}$ (in \%, blue line labelled "Error"). As can be seen, to estimate $P_{7}$ with less than $10 \%$ error, 30 additional years are needed, leading to a total horizon length of $7+30$ years. According to an investment model based on $[21,25]$ with only one week per year, an hourly basis, and a time horizon of 37 years, the execution time for such an error would be approximately $45 \mathrm{~min}$. Larger horizons lead easily to undesirable execution times or "out of memory" errors on a 64-bit Intel-Core $\mathrm{CPU}$ at 3.4 GHz. However, as the next subsection proves, the proposed truncation $C$ of $C^{\infty}$ provides the exact price $P_{7}$ with a total horizon of $7+0$ years in only $5 \mathrm{~min}$, considering the abovementioned computer.

\subsection{The valid truncation $C$ of $C^{\infty}$ for FDP}

Let us consider an annualization $f_{p, p^{\prime}}$ of $f_{p}$ in $\Gamma_{p}=\{p, \ldots, \min (p, p+l-1)\}, p \in\{0,1$, $\ldots, \underline{p}\}$ and $p^{\prime} \in \Gamma_{p}$ (thus, annualization over the generation assets' lifespan), i.e.,:

$$
f_{p}=\sum_{p^{\prime} \in \Gamma_{p}} \frac{f_{p, p^{\prime}}}{(1+r)^{p^{\prime}-p}}
$$

and such that $f_{p, p^{\prime}}$ is uniform and proportional respect to $f_{p}$, i.e.,:

$$
f_{p, p^{\prime}}=\frac{f_{p}}{s} \cdot(1+i)^{p^{\prime}-p} p \in\{0, \ldots, \underline{p}\}, p^{\prime} \in \Gamma_{p}
$$

The objective function (8) truncated to $\{0,1, \ldots, \underline{p}\}$ using $f_{p, p^{\prime}}$ becomes (taxes can be easily included by substituting $f_{p}$ by $f^{\prime} p$, as it is described in Appendix D): 


$$
C=\sum_{p=0}^{\underline{p}}\left\{K_{p} \cdot \sum_{p^{\prime} \in \Gamma_{p}} \frac{f_{p, p^{\prime}}}{(1+r)^{p^{\prime}}}\right\}
$$

Appendix F proves that the dual variables $L_{p}$ of (9) when minimizing the objective function (14) are:

$$
L_{p}=\left\{\begin{array}{c}
\frac{f_{0}}{s \cdot e} \cdot \sum_{u=p}^{p}\left(\frac{1+i}{1+r}\right)^{u} \\
\frac{f_{0}}{s \cdot e} \cdot \sum_{u=p+1}^{\left.\frac{p}{1+r}\right)^{u}}
\end{array}\right\}
$$

Using the same inputs of Table 1, in Figure 2, grey columns represent each summand in (15), positive columns (grey columns named P) corresponding to the first summation while negatives (grey columns named $\mathrm{N}$ ) to the second one. It also represents $L_{7}$ (green discontinuous line named L7) and the discounted price $P_{7} /(1+r)^{7}$ (brown line named P7) of (6), both as a function of $p$.

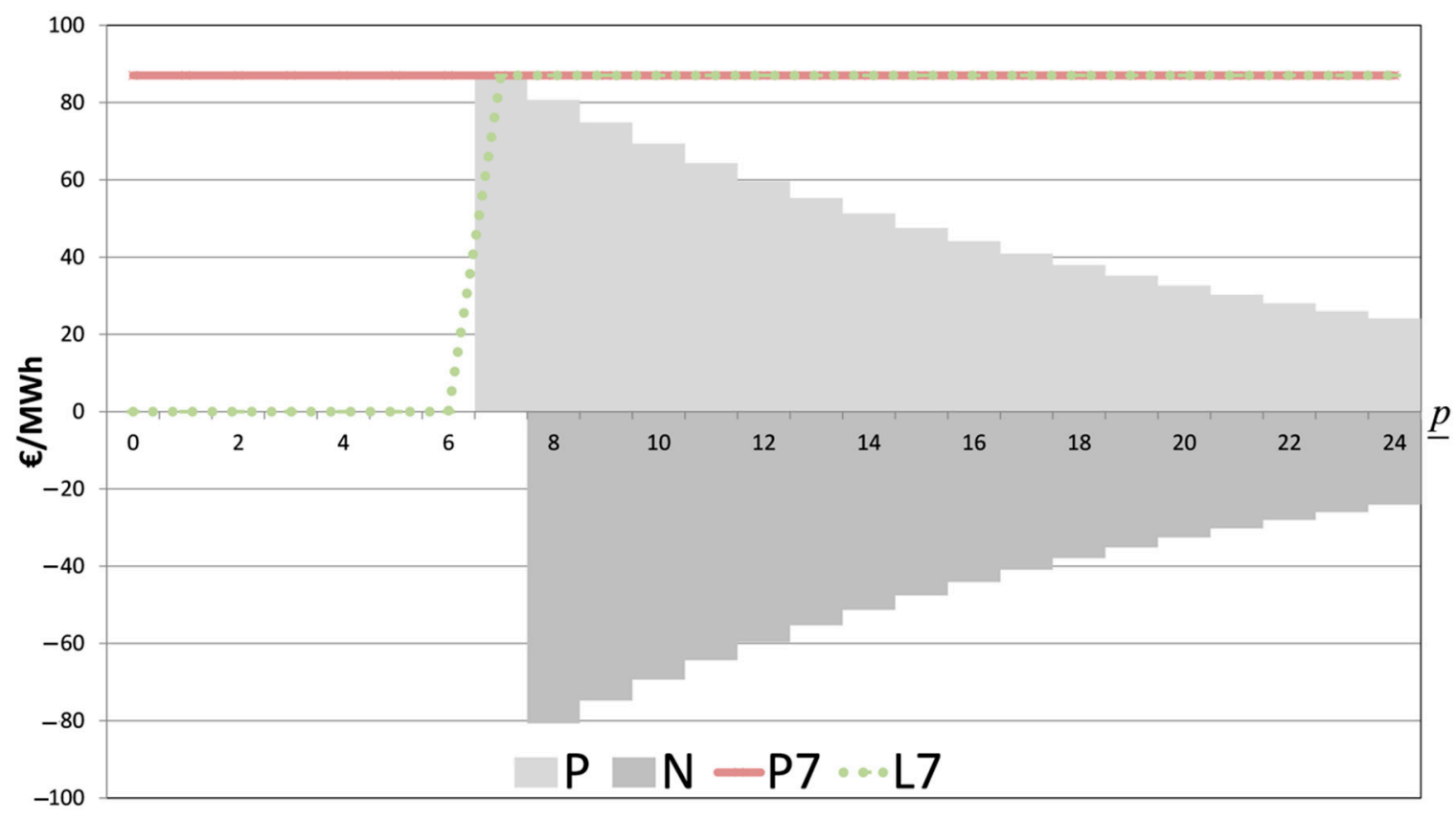

Figure 2. $C$ is a valid truncation of $\mathrm{C}^{\infty}$ for FDP.

As can be seen only the first term in the first summand of (15) is not compensated with a term in the second summand. Therefore:

$$
L_{p}=\frac{f_{0}}{s \cdot e} \cdot\left(\frac{1+i}{1+r}\right)^{p}=\frac{f_{p}}{s \cdot e \cdot(1+r)^{p}}=\frac{P_{p}}{(1+r)^{p}}
$$

Thus, $L_{p}$ are the discounted prices $P_{p}$ of (6), and no approximation is needed, i.e.:

$$
L_{\frac{p}{p}}^{p} \neq L_{p}^{\infty}=L_{p}=\frac{P_{p}}{(1+r)^{p}}
$$

For generalization purposes, proof of Appendix $\mathrm{F}$ considers the next generalization $f_{p, p}^{a}$ of $f_{p, p^{\prime}}$ (generalization if $a=i$ ) as an alternative non-uniform annualization of $f_{p}$ in $\Gamma_{p}$ for FDP, which depends on a parameter $a$ : 


$$
f_{p, p^{\prime}}^{a}=\frac{f_{p} \cdot s_{p}^{a}}{s} \cdot(1+a)^{p^{\prime}-p} p \in\{0, \ldots, \underline{p}\}, p^{\prime} \in \Gamma_{p}
$$

being:

$$
s_{p}^{a}=\frac{\sum_{p^{\prime} \in \Gamma_{p}}\left(\frac{1+i}{1+r}\right)^{p^{\prime}-p}}{\sum_{p^{\prime} \in \Gamma_{p}}\left(\frac{1+a}{1+r}\right)^{p^{\prime}-p}}
$$

\section{Conclusions}

The current context of decarbonization of the energy system, with the closing of fossil fuel plants and the electrification of energy end usages, is entailing a significant increase in generation capacity based on renewable sources of energy, most of them with negligible operation costs. In this context, there is a need to assess how the electricity prices that would allow the recovery of the total generation cost (operation and investment costs) could evolve in the future years. This paper addresses this challenge when no scarcity prices (that appear in hours with non-supplied demand) are allowed, which is the practical approach of regulators and system operators of many power systems.

Based on a stylized cost-minimization model that only considers one renewable technology for investment, this paper proposes a novel annualization of the investment costs (summarized in Equations (12) and (13)) that ensures the recovery of the investment costs. Although mathematically based on a marginalist approach with no additional capacity mechanisms, the proposed model is intended to provide the total electricity price estimation independently of the market mechanism in place. The model can be solved with a finite MP problem despite the hypothesis of infinite life for companies, and the electricity prices are obtained from the dual variables of the demand balance constraints.

With regard to other similar approaches in the literature, this approach:

- Focuses on the electricity prices instead of on the estimation of the primal investment variables, proving they are the same as those that had been obtained from the original infinite model. In addition, no residual values to represent costs in periods beyond the finite horizon are needed.

- $\quad$ Allows computing the exact $m$ marginal prices of the infinite model using the proposed model with a finite horizon of only $m$ periods. The straightforward truncation of the infinite problems would need a much longer time horizon to reach a reasonable approximation of these same prices.

Although some of the hypotheses of the model might seem simplistic (especially the assumption of a single renewable technology for investment), the proposed annualization of the investment costs must be understood as a sound financial mechanism to estimate long-term future electricity prices according to the awaited expansion of the renewable generation capacity. These types of studies are especially essential for generation companies when planning future investment strategies, but also for regulators to design suitable subsidies, capacity mechanisms, and energy policies, especially under the expected penetration of renewable plants.

The proposed annualization is a first step for long-term prices estimation to mitigate the approximation issues of other similar approaches that truncate the original infinite horizon. Current authors' work aims to extend this approach to represent several simultaneous technologies and generation companies, include storage facilities, and include non-constant utilization factors and inflation rates.

Author Contributions: Conceptualization, F.A.C.; Formal analysis, F.A.C.; Funding acquisition, J.V.; Investigation, F.A.C. and J.V.; Methodology, F.A.C. and J.V.; Project administration, F.A.C. and J.V.; Supervision, F.A.C. and E.C.; Writing-original draft, F.A.C.; Writing-review \& editing, F.A.C., J.V. and E.C. All authors have read and agreed to the published version of the manuscript.

Funding: This work has been partially financed by National Funds through the Portuguese funding agency, FCT—Fundação para a Ciência e a Tecnologia—within project UIDB/50014/2020. 
Informed Consent Statement: Not applicable.

Conflicts of Interest: The authors declare no conflict of interest.

\section{Nomenclature}

$\underline{\text { Indexes and sets }}$
$\mathbb{Z}, \mathbb{N}$
$\Gamma$
$p, \underline{p}$
$\Gamma_{\pi}$
$\underline{\text { Scalars }}$
$i$
$l$
$\underline{\text { Parameters }}$
$f_{p}$
tax
$f_{p}^{\prime}$
$d_{p}$
$r_{p}$
$e_{p}$
$k_{p}$
$f_{p, p^{\prime}}$
$f_{p, p^{\prime}}^{a}$
$a_{p}$
$\frac{\text { Variables (capital letters) }}{C^{¥}}$
$C_{-}^{p}$
$C$
$L_{p}^{¥}, L_{-p}^{p}, L_{p}$
$K_{p}$
$\Delta \Delta_{\pi}$
$P_{p}$

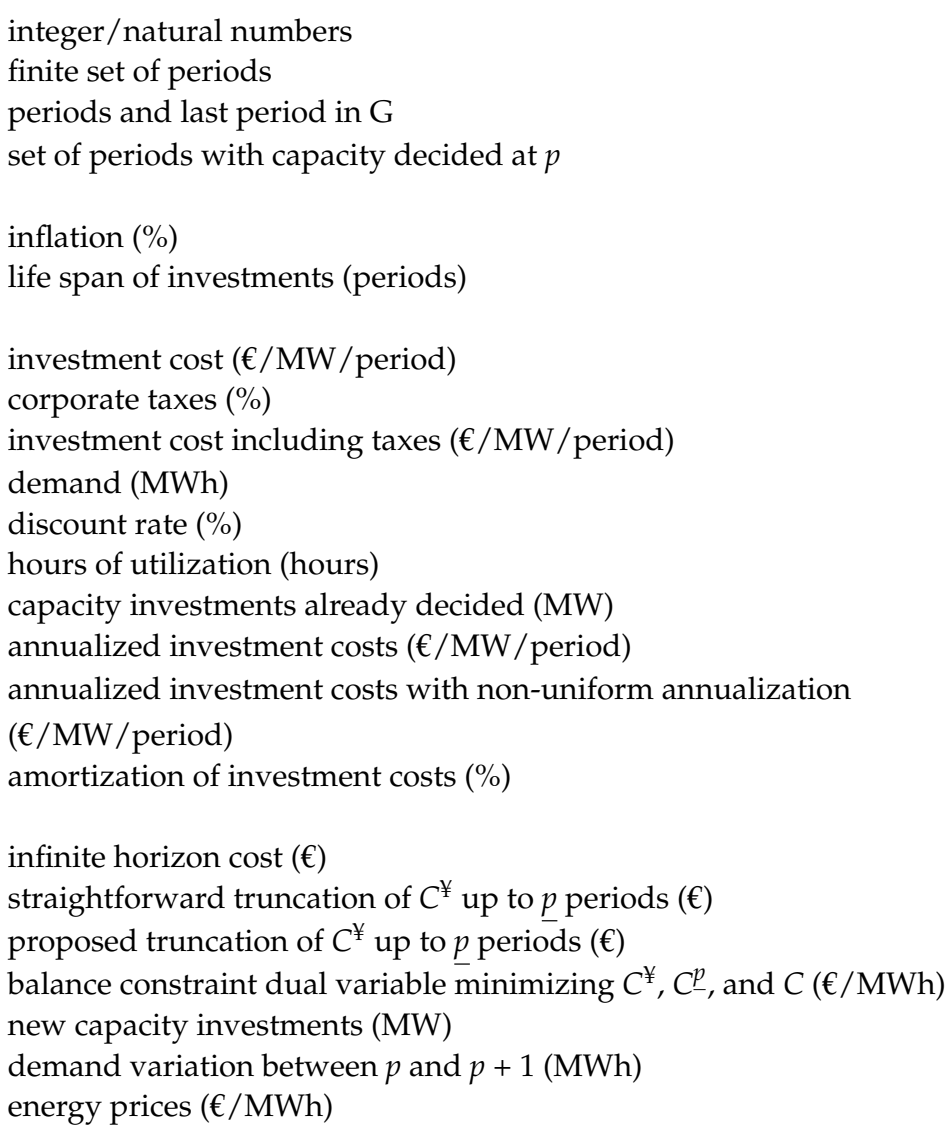

\section{Appendix A. OIP Computation}

This subsection proves that, with the above assumptions, the OIP is unique and can be computed from inputs by directly solving algebraic equations. Since $d_{p}$ must be satisfied with a utilization $e$ of the non-expired investments $K_{p^{\prime}}$ installed at periods $p^{\prime}$ previous to $p$, i.e., $p-l+1 \leq p^{\prime} \leq p$, it is:

$$
d_{p}=e \cdot \sum_{p^{\prime}=p-l+1}^{p} K_{p^{\prime}}
$$

Applying (A1) to $p-1$ and subtracting it from (A1):

$$
d_{p}-d_{p-1}=e \cdot\left(K_{p}-K_{p-l}\right)
$$

And therefore:

$$
K_{p}=K_{p-l}+\frac{d_{p}-d_{p-1}}{e}
$$

$K_{p}$ is thus given by the reposition of the capacity $K_{p-l}$ just expired (no longer available at period $p$ ) plus the amount needed to satisfy the demand variation $d_{p}-d_{p-1}$ (scaled by the utilization $e$ ). Since we are assuming that $K_{p} \geq 0$, clearing from (A3), the demand $d_{p}$ must satisfy:

$$
-\left(d_{p}-d_{p-1}\right) \leq e \cdot K_{p-l}
$$


Therefore, we add the hypothesis that $-\left(d_{p}-d_{p-1}\right)$ must not be larger than the used capacity $e \cdot K_{p-l}$ just expired, even if the demand $d_{p}$ between two consecutive periods decreases (i.e., $d_{p} \leq d_{p-1}$ ).

Applying (A5) to $p=0$, it can be seen that $K_{0}$ depends on $d_{0}$ and $d_{-1}$, but also on $k_{-l}$ which is the capacity already installed $l$ periods before the first one $p=0$. Therefore, $K_{0}$ depends only on the inputs $d_{0}, d_{-1}$, and $k_{-l}$. Analogously, for a general $p \in \mathbb{N}$, applying (A6) recursively until a period $p^{\prime} \in \Gamma \backslash \mathbb{N}$ is reached, it can be seen that $K_{p}$ is a function of inputs at periods lesser than or equal to $p$. Indeed:

$$
\begin{aligned}
& K_{p}=K_{p-l}+\frac{d_{p}-d_{p-1}}{e}=K_{p-2 \cdot l}+\frac{d_{p-l}-d_{p-l-1}}{e}+\frac{d_{p}-d_{p-1}}{e} \\
& =k_{p-m_{p} \cdot l}+\frac{d_{p-(m p-1) \cdot}-d_{p-\left(m_{p}-1\right) \cdot l-1}}{e}+\ldots+\frac{d_{p-l}-d_{p-l-1}}{e}+\frac{d_{p}-d_{p-1}}{e}
\end{aligned}
$$

where:

$$
m_{p}=\min \{m \in \mathbb{N} / p-m \cdot l<0\}
$$

\section{Appendix B. OIP Sensitivity with Respect to the Demand}

This subsection obtains some mathematical relationships between the variation $\Delta D_{p}$ in the demand $d_{p}$ at period $p$, and the variation $\Delta K_{p^{\prime}}$ of the OIP. Any variation $\Delta D_{p}$ not only affects $K_{p}$ but also $K_{p+1}$ since, applying (3) for $p+1$ :

$$
\begin{aligned}
& K_{p+1}=k_{p-m^{p+1 \cdot l}}+\frac{d_{(p+1)-\left(m^{p+1}-1\right) \cdot l}-d_{(p+1)-\left(m^{p+1}-1\right) \cdot l-1}}{e}+\ldots+ \\
& \frac{d_{(p+1)-l}-d_{(p+1)-l-1}}{e}+\frac{d_{p+1}-d_{p}}{e}
\end{aligned}
$$

Note that since $l>1$ only one term in (A7) includes $d_{p}$ (the last one). Furthermore, applying (3) to $p+n \cdot l, n \in \mathbb{N}, \Delta D_{p}$ also affects future investments $K_{p+n \cdot l}$ :

$$
\begin{aligned}
& K_{p+n \cdot l}=k_{p-m^{p+n \cdot l \cdot l}}+\frac{d_{(p+n \cdot l)-\left(m^{p+n \cdot l}-1\right) \cdot l}-d_{(p+n \cdot l)-\left(m^{p+n \cdot l}-1\right) \cdot l-1}}{e}+\ldots+ \\
& \frac{d_{p}-d_{p-1}}{e}+\ldots+\frac{d_{(p+n \cdot l)-l}-d_{(p+n \cdot l)-l-1}}{e}+\frac{d_{p+n \cdot l}-d_{(p+n \cdot l)-1}}{e}
\end{aligned}
$$

Note that (A8) is a generalization of (3) (since (3) is (A8) for $n=0$ ). Analogously, generalizing (A7), $\Delta D_{p}$ also affects $K_{p+n \cdot l+1}, n \in \mathbb{N}$ :

$$
\begin{aligned}
& K_{p+n \cdot l+1}=k_{p-m^{p+n \cdot l+1} \cdot l}+\frac{d_{(p+n \cdot l+1)-\left(m^{p+n \cdot l+1}-1\right) \cdot l}-d_{(p+n \cdot l+1)-\left(m^{p+n \cdot l+1}-1\right) \cdot l-1}}{e}+\ldots+ \\
& \frac{d_{p+1}-d_{p}}{e}+\ldots+\frac{d_{(p+n \cdot l+1)-l}-d_{(p+n \cdot l)-l}}{e}+\frac{d_{p+n \cdot l+1}-d_{p+n \cdot l}}{e}
\end{aligned}
$$

From (A8) and (A9), it is proven that for $n \in \mathbb{N}$ :

$$
\frac{\Delta K_{p^{\prime}}}{\Delta D_{p}}=\left\{\begin{array}{c}
\frac{1}{e} \text { if } p^{\prime}=p+n \cdot l \\
-\frac{1}{e} \text { if } p^{\prime}=p+n \cdot l+1 \\
0 \text { otherwise }
\end{array}\right.
$$

\section{Appendix C. Prices to Recover Investment Costs}

Prices that recover investment costs $f_{p^{\prime}}$ over the generation assets' lifespan should satisfy:

$$
f_{p^{\prime}}=\sum_{\tau=0}^{l-1} \frac{e \cdot P_{p^{\prime}+\tau}}{(1+r)^{\tau}}
$$

Note that $f_{p^{\prime}}$ is assumed to be satisfied with prices $P_{p}$ in years $p$ in the lifespan of the capacity installed at $p^{\prime}$. Applying (A11) to $p^{\prime}=p+n \cdot l$ and $p^{\prime}=p+n \cdot l+1, n \in \mathbb{N}$, dividing the later by $(1+r)$, and subtracting both: 


$$
f_{p+n \cdot l}-\frac{f_{p+n \cdot l+1}}{(1+r)}=e \cdot P_{p+n \cdot l}-\frac{e \cdot P_{p+(n+1) \cdot l}}{(1+r)^{l}}
$$

Applying (1) to $f_{p+n \cdot n^{\cdot} l+1}$ in (A12) and then to $f_{p+n} \cdot{ }^{\cdot}$ :

$$
f_{p+n \cdot l} \cdot\left(1-\frac{1+i}{1+r}\right)=e \cdot\left(P_{p+n \cdot l}-\frac{P_{p+(n+1) \cdot l}}{(1+r)^{l}}\right) \Leftrightarrow \frac{f_{p}}{e} \cdot(1+i)^{n \cdot l} \cdot\left(\frac{r-i}{1+r}\right)=P_{p+n \cdot l}-\frac{P_{p+(n+1) \cdot l}}{(1+r)^{l}}
$$

Dividing (A13) by $(1+r)^{n \cdot l}$ :

$$
\frac{f_{p}}{e} \cdot\left(\frac{1+i}{1+r}\right)^{n \cdot l} \cdot\left(\frac{r-i}{1+r}\right)=\frac{P_{p+n \cdot l}}{(1+r)^{n \cdot l}}-\frac{P_{p+(n+1) \cdot l}}{(1+r)^{(n+1) \cdot l}}
$$

Note that according to(A14), for any $k \in \mathbb{N}$ :

$$
\sum_{n=0}^{k}\left\{\frac{f_{p}}{e} \cdot\left(\frac{1+i}{1+r}\right)^{n \cdot l} \cdot\left(\frac{r-i}{1+r}\right)\right\}=P_{p}-\frac{P_{p+(k+1) \cdot l}}{(1+r)^{(k+1) \cdot l}}
$$

Therefore, taking limits in $k$ :

$$
\frac{f_{p}}{e} \cdot\left(\frac{r-i}{1+r}\right) \cdot \sum_{n=0}^{\infty}\left(\frac{1+i}{1+r}\right)^{n \cdot l}=P_{p}-\lim _{k \rightarrow \infty} \frac{P_{p+(k+1) \cdot l}}{(1+r)^{(k+1) \cdot l}}
$$

Denoting $\operatorname{Lim}_{p}$ as the above limit and clearing $P_{p},(\mathrm{~A} 16)$ is:

$$
P_{p}=\frac{f_{p}}{e \cdot s}+\operatorname{Lim}_{p}
$$

where:

$$
s=\frac{1}{\left(\frac{r-i}{1+r}\right) \cdot \sum_{n=0}^{\infty}\left(\frac{1+i}{1+r}\right)^{n \cdot l}}=\frac{1-\left(\frac{1+i}{1+d}\right)^{l}}{\left(\frac{r-i}{1+r}\right)}=\sum_{p^{\prime}=0}^{l-1}\left(\frac{1+i}{1+r}\right)^{p^{\prime}}
$$

which converges if $i<r$ (see Section 2). Note that parameter $s$ is a function of RDF.

Substituting (A17) in (A11), and taking into account (1) and (A18):

$$
f_{p}=\sum_{\tau=0}^{l-1}\left[\frac{e}{(1+r)^{\tau}} \cdot\left(\frac{f_{p+\tau}}{e \cdot s}+\operatorname{Lim}_{p+\tau}\right)\right]=f_{p} \cdot \frac{1}{s} \cdot \sum_{\tau=0}^{l-1}\left(\frac{1+i}{1+r}\right)^{\tau}+e \cdot \sum_{\tau=0}^{l-1} \frac{\operatorname{Lim}_{p+\tau}}{(1+r)^{\tau}}=f_{p}+e \cdot \sum_{\tau=0}^{l-1} \frac{\operatorname{Lim}_{p+\tau}}{(1+r)^{\tau}}
$$

Therefore:

$$
\sum_{\tau=0}^{l-1} \frac{\operatorname{Lim}_{p+\tau}}{(1+r)^{\tau}}=0
$$

Since $\operatorname{Lim}_{p} \geq 0$, (A20) holds if and only if $\operatorname{Lim}_{p}$ is 0 for every period $p$. Therefore, from (1) and (A17), prices to recover investment costs satisfy (6).

\section{Appendix D. Considering Corporate Taxes}

This annex adapts (6) when there are corporate taxes charged on the profits generated from the exploitation of the investments. Profits are computed by subtracting book amortizations from incomes, that is, from the allocation of the investment cost $f_{p^{\prime}}$ over the periods $p^{\prime}$ in which the assets are used.

Let us consider that the amortization period coincides with the lifespan of an asset and that the amortization is represented through a percentage $a_{\tau+p}$ of $f_{p}$ for the $l$ periods $\tau$ starting from $p, \tau \in\{0, \ldots, l-1\}$. Then: 


$$
\sum_{\tau=0}^{l-1} a_{p+\tau}=1
$$

Similarly to (A11), to recover investment costs, prices $P_{p}$ must satisfy:

$$
f_{p}+\operatorname{tax} \cdot\left(\sum_{\tau=0}^{l-1} \frac{e \cdot P_{p+\tau}}{(1+r)^{\tau}}-\sum_{\tau=0}^{l-1} \frac{f_{p} \cdot a_{p+\tau}}{(1+r)^{\tau}}\right)=\sum_{\tau=0}^{l-1} \frac{e \cdot P_{p+\tau}}{(1+r)^{\tau}}
$$

being tax the percentage of taxes applied to the profit, assuming that incomes are greater than or equal to the amortizations. Extracting common factor to the right-hand side of A22) and clearing, Equation (A22) is equivalent to:

$$
f_{p} \cdot\left(\frac{1-\operatorname{tax} \cdot c a_{p}}{1-\operatorname{tax}}\right)=\sum_{\tau=0}^{l-1} \frac{e \cdot P_{p+\tau}}{(1+r)^{\tau}}
$$

where $c a_{p}$ is the present value, at period $p$, of the summation of the percentages $a_{\tau+p}$ for $\tau=0, \ldots, l-1$, i.e.,:

$$
c a_{p}=\sum_{\tau=0}^{l-1} \frac{a_{p+\tau}}{(1+r)^{\tau}}
$$

Note that, according to(A21), $c a_{p}<1$ and therefore the left-hand side of (A23) is higher than $f_{p}$, which can be interpreted as the investment $\operatorname{cost} f^{\prime} p$ including the effect of taxes, i.e.,:

$$
f_{p}^{\prime}=f_{p} \cdot\left(\frac{1-\operatorname{tax} \cdot c a_{p}}{1-\operatorname{tax}}\right)
$$

Following the same reasoning as in the previous subsection, but starting from (A23) and (A25) instead of (A11), the prices $P_{p}^{\prime}$ taking taxes into account can now be computed as in (6) but using $f_{p}^{\prime}$ instead of $f_{p}$.

\section{Appendix E. Prices to Recover Investment Costs as Dual Variables of $\mathbf{C}^{\infty}$}

By definition, $L_{p}^{\infty}$ is:

$$
L_{p}^{\infty}=\lim _{\Delta D_{p} \rightarrow 0} \frac{\Delta C^{\infty}}{\Delta D_{p}}=\sum_{p^{\prime}=0}^{\infty} \frac{f_{p^{\prime}} \cdot \lim _{\Delta D_{p} \rightarrow 0}\left(\Delta K_{p^{\prime}} / \Delta D_{p}\right)}{(1+r)^{p^{\prime}}}
$$

According to (5) (note that $\Delta K_{p^{\prime}} / \Delta D_{p}$ is independent of $\Delta D_{p}$ ):

$$
L_{p}^{\infty}=\left\{\begin{array}{c}
\sum_{n=0}^{\infty} \frac{f_{p+n \cdot l \cdot} \lim _{\Delta D p \rightarrow 0}\left(\frac{\Delta K_{p+n \cdot l}}{\Delta D p}\right)}{(1+r)^{p+n \cdot l}} \\
+ \\
\sum_{n=0}^{\infty} \frac{f_{p+n \cdot l+1} \cdot \lim _{\Delta D p \rightarrow 0}\left(\frac{\Delta K_{p+n \cdot l+1}}{\Delta D p}\right)}{(1+r)^{p+n \cdot l+1}}
\end{array}\right\}=\left\{\sum_{n=0}^{\infty} \frac{f_{p+n \cdot l} \cdot(1 / e)}{(1+r)^{p+n \cdot l}}-\sum_{n=0}^{\infty} \frac{f_{p+n \cdot l+1} \cdot(1 / e)}{(1+r)^{p+n \cdot l+1}}\right\}
$$

and, therefore, (1), (A18), and (6) leads to:

$$
L_{p}^{\infty}=\left\{\begin{array}{c}
\sum_{n \in\{\mathbb{N}: p+n \cdot l \leq \infty\}} \frac{f_{0}}{e} \cdot\left(\frac{1+i}{1+r}\right)^{p+n \cdot l} \\
\sum_{n \in\{\mathbb{N}: p+n \cdot l+1 \leq \infty\}} \frac{f_{0}}{e} \cdot\left(\frac{1+i}{1+r}\right)^{p+n \cdot l+1}
\end{array}\right\}
$$

and finally: 


$$
\begin{aligned}
& L_{p}^{\infty}=\frac{f_{0}}{e} \cdot\left(\frac{1+i}{1+r}\right)^{p} \cdot\left(\frac{r-i}{1+r}\right) \cdot \sum_{n=0}^{\infty}\left(\frac{1+i}{1+r}\right)^{n \cdot l} \\
& =\frac{f_{0}}{e} \cdot\left(\frac{1+i}{1+r}\right)^{p} \cdot \frac{1}{s}=\frac{f_{p}}{s \cdot e} \cdot \frac{1}{(1+r)^{p}}=\frac{P_{p}}{(1+r)^{p}}
\end{aligned}
$$

This last equation proves that $L^{\infty}$ is indeed the discounted price $P_{p}$ of (6).

\section{Appendix F. Prices to Recover Investment Costs as Dual Variables of $C$ with FDP}

The dual variable $L_{p}$ of (9) when minimizing $C$ using $f_{p, p}^{a}$ in (14) with $\Gamma_{p}=\{\underline{p}, \ldots$, $\min (p, p+l-1)\}$ :

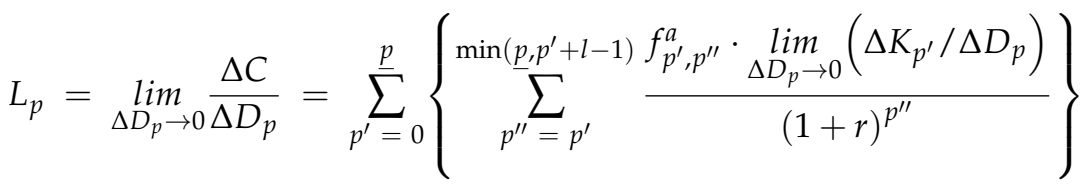

Applying now (1) and (19) (note that $\Delta K_{p^{\prime}} / \Delta D_{p}$ is independent of $\Delta D_{p}$ ):

$$
\begin{aligned}
& L_{p}=\sum_{p^{\prime}=0}^{p}\left[\frac{f_{p^{\prime}}}{(1+r)^{p^{\prime}}} \cdot \frac{s_{p^{\prime}}^{a}}{s} \cdot\left(\Delta K_{p^{\prime}} / \Delta D_{p}\right) \cdot\left\{\sum_{p^{\prime \prime}=p^{\prime}}^{\min \left(\underline{p}, p^{\prime}+l-1\right)}\left(\frac{1+a}{1+r}\right)^{p^{\prime \prime}-p^{\prime}}\right\}\right] \\
& =f_{0} \cdot \sum_{p^{\prime}=0}^{\underline{p}}\left[\left(\frac{1+i}{1+r}\right)^{p^{\prime}} \cdot \frac{s_{p^{\prime}}^{a}}{s} \cdot\left(\Delta K_{p^{\prime}} / \Delta D_{p}\right) \cdot\left\{\sum_{p^{\prime \prime}=p^{\prime}}^{\min \left(\underline{p}, p^{\prime}+l-1\right)}\left(\frac{1+a}{1+r}\right)^{p^{\prime \prime}-p^{\prime}}\right\}\right] \\
& =f_{0} \cdot \sum_{p^{\prime}=0}^{\underline{p}}\left[\left(\frac{1+i}{1+r}\right)^{p^{\prime}} \cdot \frac{1}{s} \cdot\left(\Delta K_{p^{\prime}} / \Delta D_{p}\right) \cdot\left\{\sum_{p^{\prime \prime}=p^{\prime}}^{\min \left(\underline{p}, p^{\prime}+l-1\right)}\left(\frac{1+i}{1+r}\right)^{p^{\prime \prime}-p^{\prime}}\right\}\right] \\
& =f_{0} \cdot \sum_{p^{\prime}=0}^{\underline{p}}\left[\frac { 1 } { s } \cdot ( \Delta K _ { p ^ { \prime } } / \Delta D _ { p } ) \cdot \left\{\begin{array}{c}
\min \left(\underline{p}, p^{\prime}+l-1\right) \\
\left.\left.\sum_{p^{\prime \prime}=p^{\prime}}\left(\frac{1+i}{1+r}\right)^{p^{\prime \prime}}\right\}\right]
\end{array}\right.\right.
\end{aligned}
$$

From (5):

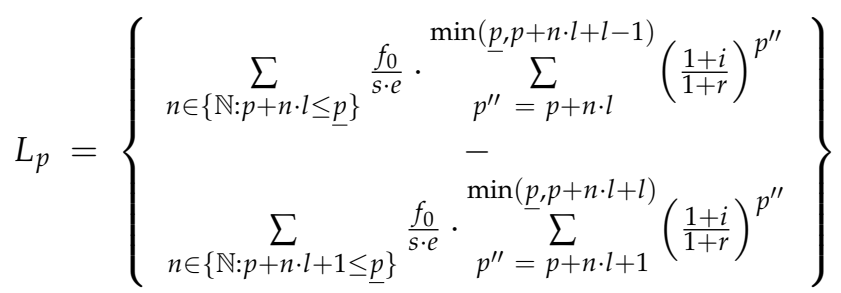

In this expression, each term except the first one (for $n=0$ ) in the first summand is the opposite of a term in the second summand. Effectively, if $n_{p}=\max \{n \in \mathbb{N}: p+n \cdot l+1 \leq \underline{p}\}$, the first summand can be reduced to:

$$
\begin{aligned}
& \sum_{n \in\{\mathbb{N}: p+n \cdot l \leq \underline{p}\} \quad \min (\underline{p}, p+n \cdot l+l-1)}^{p^{\prime \prime}=p+n \cdot l}\left(\frac{1+i}{1+r}\right)^{p^{\prime \prime}}=\sum_{n=0}^{n_{p}}\left[\sum_{p^{\prime \prime}=p+n \cdot l}^{\min (\underline{p}, p+n \cdot l+l-1)}\left(\frac{1+i}{1+r}\right)^{p^{\prime \prime}}\right]= \\
& {\left[\left(\frac{1+i}{1+r}\right)^{p}+\left(\frac{1+i}{1+r}\right)^{p+1}+\ldots+\left(\frac{1+i}{1+r}\right)^{p+l-1}\right]} \\
& +\left[\left(\frac{1+i}{1+r}\right)^{p+l}+\left(\frac{1+i}{1+r}\right)^{p+l+1}+\ldots+\left(\frac{1+i}{1+r}\right)^{p+l+l-1}\right] \\
& +\ldots \\
& +\left[\left(\frac{1+i}{1+r}\right)^{p+n_{p} \cdot l}+\left(\frac{1+i}{1+r}\right)^{p+n_{p} \cdot l+1}+\ldots+\left(\frac{1+i}{1+r}\right)^{\underline{p}}\right] \\
& =\sum_{u=p}^{p}\left(\frac{1+i}{1+r}\right)^{u}
\end{aligned}
$$


and, analogously, the second summand becomes:

$$
\sum_{n=0}^{n_{p}}\left[\sum_{p^{\prime \prime}=p+n \cdot l+1}^{\min (\underline{p}, p+n \cdot l+l)}\left(\frac{1+i}{1+r}\right)^{p^{\prime \prime}}\right]=\sum_{u=p+1}^{\underline{p}}\left(\frac{1+i}{1+r}\right)^{u}
$$

Therefore:

$$
L_{p}=\left\{\begin{array}{c}
\frac{f_{0}}{s \cdot e} \cdot \sum_{u=p}^{p}\left(\frac{1+i}{1+r}\right)^{u} \\
- \\
\frac{f_{0}}{s \cdot e} \cdot \sum_{u=p+1}^{p}\left(\frac{1+i}{1+r}\right)^{u}
\end{array}\right\}
$$

Therefore, the dual variable $L_{p}$ coincides exactly with $P_{p} /(1+r)^{p}$, the first term in the first summand (see (6)):

$$
L_{p}=\frac{f_{0}}{s \cdot e} \cdot\left(\frac{1+i}{1+r}\right)^{p}=\frac{f_{p}}{s \cdot e \cdot(1+r)^{p}}=\frac{P_{p}}{(1+r)^{p}}
$$

Note that no approximation is needed.

\section{References}

1. Pérez-Arriaga, I.J. Regulation of the Power Sector; Springer Science \& Business Media: Berlin, Germany, 2014; ISBN 978-1-4471-5034-3.

2. Genoese, F.; Drabik, E.; Egenhofer, C. The EU Power Sector Needs Long-Term Price Signals. Social Science Research Network, SSRN Scholarly Paper ID 2782355. 2016. Available online: https:/ / papers.ssrn.com/abstract=2782355 (accessed on 30 May 2019 ).

3. Mastropietro, P. Regulatory Design of Capacity Remuneration Mechanisms in Regional and Low-Carbon Electric Power Markets. Ph.D. Thesis, KTH Royal Institute of Technology, Stockholm, Sweden, 2016.

4. $\quad$ Eckaus, R.S.; Parikh, K.S. Planning for Growth; MIT Press: Cambridge, MA, USA, 1968.

5. Conejo, A.J.; Baringo, L.; Kazempour, S.J.; Siddiqui, A.S. Investment in Electricity Generation and Transmission: Decision Making under Uncertainty; Springer: Berlin/Heidelberg, Germany, 2016; ISBN 978-3-319-29501-5.

6. Ramos, A.; Perez-Arriaga, I.J.; Bogas, J. A nonlinear programming approach to optimal static generation expansion planning. IEEE Trans. Power Syst. 1989, 4, 1140-1146. [CrossRef]

7. Ventosa, M.; Denis, R.; Redondo, C. Expansion planning in electricity markets. Two different approaches. In Proceedings of the 14th Power Systems Computation Conference 2002 (PSCC 2002 SEVILLE), Sevilla, Spain, 24-28 June 2002; pp. 1-7. [CrossRef]

8. Murphy, F.H.; Smeers, Y. Generation Capacity Expansion in Imperfectly Competitive Restructured Electricity Markets. Oper. Res. 2005, 53, 646-661. [CrossRef]

9. Wang, J.; Shahidehpour, M.; Li, Z.; Botterud, A. Strategic Generation Capacity Expansion Planning With Incomplete Information. IEEE Trans. Power Syst. 2009, 24, 1002-1010. [CrossRef]

10. Kazempour, S.J.; Conejo, A.J.; Ruiz, C. Strategic Generation Investment Using a Complementarity Approach. IEEE Trans. Power Syst. 2011, 26, 940-948. [CrossRef]

11. Hinojosa-Mateus, V.H. Static generation capacity expansion planning using linear transmission distribution factors. In Proceedings of the 2016 IEEE PES Transmission Distribution Conference and Exposition-Latin America (PES T\&D-LA), Morelia, Mexico, 20-24 September 2016; pp. 1-6.

12. van Stiphout, A.; Vos, K.D.; Deconinck, G. The Impact of Operating Reserves on Investment Planning of Renewable Power Systems. IEEE Trans. Power Syst. 2017, 32, 378-388. [CrossRef]

13. Park, H.; Baldick, R. Stochastic Generation Capacity Expansion Planning Reducing Greenhouse Gas Emissions. IEEE Trans. Power Syst. 2019, 30, 1002-1010. [CrossRef]

14. Gil, E.; Aravena, I.; Cárdenas, R. Generation Capacity Expansion Planning Under Hydro Uncertainty Using Stochastic Mixed Integer Programming and Scenario Reduction. IEEE Trans. Power Syst. 2015, 30, 1838-1847. [CrossRef]

15. Shengyu, W.; Lu, C.; Xiaoqing, Y.; Bo, Y. Long-term generation expansion planning under uncertainties and fluctuations of multi-type renewables. In Proceedings of the 2015 IEEE 5th International Conference on Power Engineering, Energy and Electrical Drives (POWERENG), Riga, Latvia, 11-13 May 2015; pp. 612-616.

16. Gorenstin, B.G.; Campodonico, N.M.; Costa, J.P.; Pereira, M.V.F. Power system expansion planning under uncertainty. IEEE Trans. Power Syst. 1993, 8, 129-136. [CrossRef]

17. Domínguez, R.; Conejo, A.J.; Carrión, M. Investing in Generation Capacity: A Multi-Stage Linear-Decision-Rule Approach. IEEE Trans. Power Syst. 2016, 31, 4784-4794. [CrossRef]

18. Wogrin, S.; Barquín, J.; Centeno, E. Capacity Expansion Equilibria in Liberalized Electricity Markets: An EPEC Approach. IEEE Trans. Power Syst. 2013, 28, 1531-1539. [CrossRef] 
19. Wogrin, S.; Centeno, E.; Barquin, J. Generation Capacity Expansion in Liberalized Electricity Markets: A Stochastic MPEC Approach. IEEE Trans. Power Syst. 2011, 26, 2526-2532. [CrossRef]

20. Li, Q.; Wang, J.; Zhang, Y.; Fan, Y.; Bao, G.; Wang, X. Multi-Period Generation Expansion Planning for Sustainable Power Systems to Maximize the Utilization of Renewable Energy Sources. Sustainability 2020, 12, 1083. [CrossRef]

21. González, P.; Villar, J.; Díaz, C.A.; Campos, F.A. Joint energy and reserve markets: Current implementations and modeling trends. Electr. Power Syst. Res. 2014, 109, 101-111. [CrossRef]

22. Manne, A.S. Sufficient Conditions for Optimality in an Infinite Horizon Development Plan. Econometrica 1970, 38, 18. [CrossRef]

23. Williams, J.B. The Theory of Investment Value; Fraser Publishing Co.: Burlington, VT, USA, 1997; ISBN 978-0-87034-126-7.

24. Renewable Power Generation Costs in 2014. Available online: https://irena.org/-/media/Files/IRENA/Agency/Publication/ 2015/IRENA_RE_Power_Costs_2014_report.pdf (accessed on 1 January 2015).

25. de Sisternes, M.V.F. Investment Model for Renewable Electricity Systems (IMRES): An Electricity Generation Capacity Expansion Formulation with Unit Commitment Constraints; MIT Press: Cambridge, MA, USA, 2013. 